\title{
MÔ HÌNH ĐÁNH GIÁ SỰ PHÙ HỢP CỦA HỆ THỐNG THÔNG TIN KẾ TOÁN ẢNH HƯỞNG ĐẾN HIẸU QUẢ HOẠT ĐỘNG KINH DOANH CỦA DOANH NGHIỆP
}

\author{
TRẦ THÚ' BA, NGUYẼ̃N THI TỐ QUYÊN \\ Khoa Kế toán Kiểm toán, Trường Đại học Công nghiệp TP.HCM; \\ tranthuba@iuh.edu.vn
}

Tóm tắt. Trong bối cảnh Việt Nam đang mở cửa hội nhập với khu vực và thế giới, thì các doanh nghiệp Việt Nam có xu hướng ngày càng quan tâm hơn về việc tổ chức hệ thống thông tin kế toán nhằm nâng cao hiệu quả hoạt động kinh doanh và nâng cao năng lực cạnh tranh trên thị trường. Nhóm tác giả đã sử dụng phương pháp nghiên cứu định tính kết hợp với nghiên cứu định lượng để xây dựng mô hình đánh giá sự ảnh hưởng và mức độ đóng góp của các yếu tố nhu cầu thông tin và khả năng đáp ứng nhu cầu thông tin trong sự phù hợp của hệ thống thông tin kế toán. Đo lường và đánh giá mức độ ảnh hưởng của sự phù hợp trong hệ thống thông tin kế toán đển hiệu quả hoạt động kinh doanh của doanh nghiệp tại Việt Nam. Kết quả nghiên cứu thực nghiệm với 232 doanh nghiệp đang hoạt động tại khu vực kinh tế trọng điểm: TP. Hồ Chí Minh, Đồng Nai, Bình Dương, Bà Rịa Vũng tàu, Long An, Tây Ninh cho thấy các nhân tố là nhu cầu thông tin và khả năng đáp ứng nhu cầu thông tin đều có ảnh hưởng và đóng góp tích cực vào sự phù hợp của hệ thống thông tin kế toán, cuối cùng sự phù hợp của Hệ thống thông tin kế toán tác động mạnh đến hiệu quả hoạt động kinh doanh của doanh nghiệp. Từ đó, nhóm tác giả đưa ra một số gợi ý về định hướng xây dựng và phát triển hệ thống thông tin kế toán có khả năng xử lý và đáp ứng được nhu cầu thông tin của các nhà quản lý doanh nghiệp, góp phần nâng cao hiệu quả hoạt động kinh doanh của doanh nghiệp Việt Nam trong giai đoạn hội nhập.

Từ khóa. Hiệu quả hoạt động kinh doanh, hệ thống thông tin kế toán, nhân tố tác động

\section{MODEL EVALUATE ALIGNMENT OF ACCOUNTING INFORMATION SYSTEM EFFECT TO FIRM PERFORMANCE}

\begin{abstract}
In the context of Vietnam is opening to integrate with the region and the world, most of Vietnamese enterprises have a trend more and more to consider much more about the Accounting information system (AIS) to enhance the firm performance (P) and competitive ability of firm on the market. The authors had used the qualitative methods combined the quantitative methods to research building the model evaluate the impact and levels of contribution of factors - information request (IR) and information capacity (IC) meet IR in alignment of accounting information system (AIS). Measuring and evaluating level of impact of AIS alignment to the bussines efficiency of firm. The research results with 232 enterpries have been operating at focal-point economic areas of Vietnamese south: Ho Chi Minh City, provinces: Dong Nai, Binh Duong, Ba Ria Vung Tau, Long An, Tay Ninh showed that factors are IR and IC either affected \& contributed positively to AIS alignment, finally AIS alignment impacted strongly to firm performance (P). Since then, the authors give some suggestion about the orientation of building and developing the Accounting information system that can handle and meet Administrators' information demands, contributing to enhance firm performance of Vietnam enterprises in the integration period.
\end{abstract}

Keywords. firm performance, accounting information system, impact factor.

\section{GIỚI THIỆU}

Ngày nay với sự phát triển của công nghệ thông tin đã góp phần cung cấp thông tin kịp thời, đáng tin cậy, nhanh chóng, giúp cho các nhà quản lý doanh nghiệp xử lý các công việc nhanh chóng và hiệu quả. Kế toán là một bộ phận quan trọng của hệ thống quản lý, có vai trò tích cực trong việc quản lý điều hành 
và kiểm soát các hoạt động sản xuất, kinh doanh của doanh nghiệp. Hệ thống thông tin kế toán (AIS) cung cấp thông tin kế toán, tài chính, cho nhà quản lý các cấp trong doanh nghiệp, hội đồng quản trị và những người sử dụng bên ngoài doanh nghiệp để họ ra quyết định phù hợp. Chính vì vậy Hệ thống thông tin kế toán là một nhân tố không thể thiếu trong hệ thống các công cụ quản lý doanh nghiệp hiệu quả. Nghiên cứu này đã chỉ ra rằng mức độ phù hợp của Hệ thống thông tin kế toán phụ thuộc vào năng lực cung cấp thông tin của AIS và các nhu cầu thông tin từ AIS. Một hệ thống thông tin kế toán càng phù hợp sẽ giúp cho hoạt động sản xuất kinh doanh của doanh nghiệp càng hiệu quả.

\section{CƠ SỞ LÝ THUYẾT VÀ MÔ HİNH NGHIÊN CÚ̉}

Theo lý thuyết xử lý thông tin (Galbraith, 1973) thì khả năng xử lý thông tin cần phải phù hợp với các nhu cầu thông tin của tổ chức thì nó sẽ tác động đáng kể đến hiệu quả hoạt động của tổ chức. Theo lý thuyết này, hiệu quả của khả năng xử lý thông tin được đánh giá phụ thuộc vào nhu cầu thông tin của tổ chức. Khi các công ty có khả năng xử lý thông tin có thể đáp ứng được nhu cầu thông tin thì sự phù hợp này sẽ có tác động đáng kể đến hiệu quả hoạt động của tổ chức. El Louadi (1998) và Khazanchi (2005), Ismail \& King (2005) đã áp dụng thành công lý thuyết này trong các tổ chức vừa và nhỏ và cách tiếp cận này cũng phù hợp với đề xuất của Van de Ven \& Drazin's (1984) rằng kết quả của tổ chức là sự phù hợp giữa hai hay nhiều yếu tố. Đồng thời lý thuyết xử lý thông tin của Galbraith đề cập đến nhu cầu thông tin và khả năng xử lý thông tin của tổ chức thì Ismail \& King (2005) đã chú trọng vào sự phù hợp giữa các yêu cầu thông tin kế toán và khả năng xử lý của hệ thống thông tin dựa trên máy tính để tạo ra thông tin của hệ thống thông tin kế toán. Qua việc tổng hợp các nghiên cứu trước đây như: Abernethy \& Guthrie (1994), Cragg et al., (2002), Ismail \& King (2005), Gul (1991), Raymond (1995), Fuller (1996), Chan et al (1997). Nhóm tác giả xác định hướng phát triển nghiên cúu của mô hình và đề xuất mô hình nghiên cứu dựa trên nghiên cứu của Ismail \& King (2005). Khảo sát của Ismail \& King (2005) dựa trên bốn biến. Với hai biến trung gian là các nhu cầu thông tin kế toán đối với hệ thống thông tin kế toán và khả năng đáp ưng nhu cầu thông tin của hệ thống thông tin kế toán; một biến độc lập là sự phù hợp của hệ thống thông tin kế toán; một biến phu thuộc là hiệu quả hoạt động kinh doanh. Đề tài nghiên cứu này sẽ vận dụng mô hình nghiên cứu của Ismail \& King (2005) trong bối cảnh tại Việt Nam - khu vục kinh tế trọng điểm phía nam. Để khẳng định các nhân tố trong sự phù hợp của hệ thống thông tin kế toán, và tác động của sự phù hợp trong hệ thống thông tin kế toán đến hiệu quả hoạt động kinh doanh, nhóm tác giả tiến hành phỏng vấn các chuyên gia trong lĩnh vực hệ thống thông tin kế toán. Mục đích của cuộc phỏng vấn nhằm xem xét khả năng hợp lý của việc vận dụng mô hình Ismail \& King (2005) vào môi trường doanh nghiệp Việt Nam. Kết quả của nghiên cứu sơ bộ này sẽ là cơ sở để thiết kế bảng câu hỏi cho nghiên cứu chính thức.

Hơn nữa, các nhu cầu về thông tin kế toán đối với hệ thống thông tin kế toán thì không có mối quan hệ trực tiếp với hiệu quả hoạt động. Đồng thời không có mối quan hệ trực tiếp giữa khả năng đáp ứng thông tin của hệ thống thông tin kế toán với hiệu quả hoạt động. Bất kỳ mối quan hệ nào giữa các nhu cầu thông tin kế toán hoạc khả năng đáp ứng của hệ thống thông tin kế toán đến hiệu quả hoạt động đều thông qua sư phù hợp của hệ thống thông tin kế toán. Sự phù hợp của hệ thống thông tin kế toán ảnh hưởng trực tiếp đến hiệu quả hoạt động của tổ chức (Ismail \& King, 2005). Trên cơ sở lý thuyết nghiên cứu nhóm tác giả đã đề xuất mô hình nghiên cứu như sau (hình 1). 


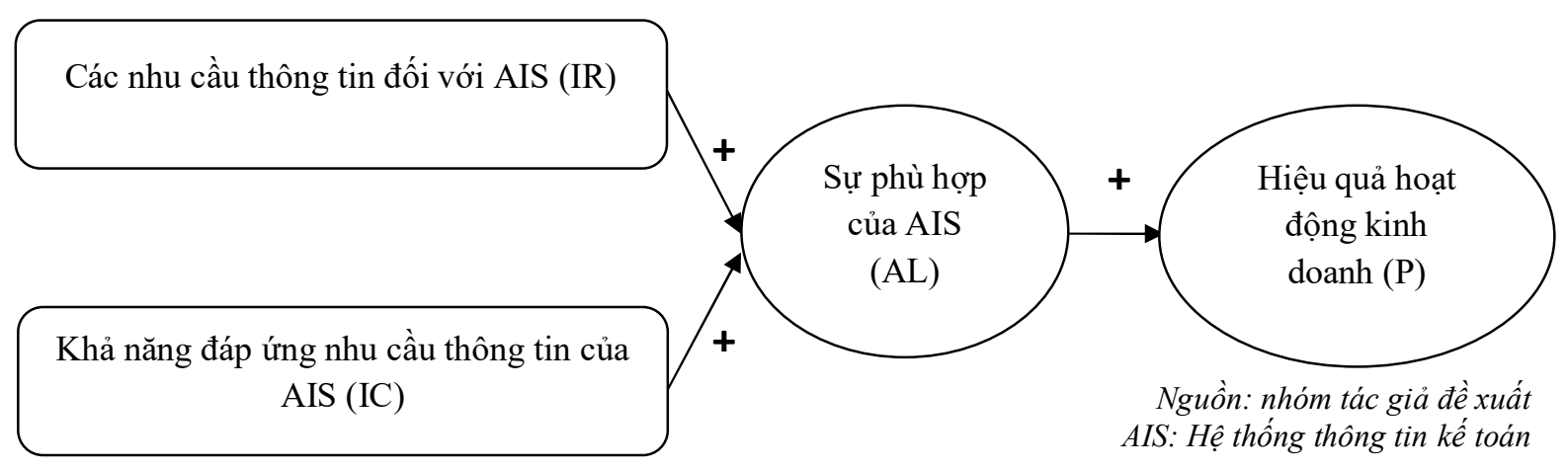

Hình 1. Mô hình nghiên cứu đề xuất

Trong mô hình nghiên cứu trên gồm 4 nhân tố là Nhu cầu thông tin đối với hệ thống thông tin kế toán (AIS requirements - IR), Khả năng đáp ưng nhu cầu thông tin của hệ thống thông tin kế toán (AIS capacity -IC), Sự phù hơp của hệ thống thông tin kế toán (AIS alignment-AL), Hiệu quả hoạt động kinh doanh (Performance - P).

\section{Một số khái niệm cơ bản}

Hiệu quả hoạt động kinh doanh: là một phạm trù kinh tế phản ánh trình độ sử dụng các nguồn lực để đạt được mục tiêu đã đặt ra, nó biểu hiện mối quan hệ giữa kết quả đạt được và những chi phí bỏ ra để có được kết quả đó, độ chênh lệch giữa hai đại lượng này càng lớn thì hiệu quả càng cao. Trên góc độ này thì hiệu quả đồng nhất với lợi nhuận của doanh nghiệp và khả năng đáp ứng về mặt chất lượng của sản phẩm đối với nhu cầu của thị trường [Ngô Đình Giao (1997) \& Nguyễn Thị Lan Hương (2013)].

Sư phù hợp của hệ thống thông tin kế toán: là mức độ phù hợp giữa các nhu cầu thông tin kế toán của người sử dụng và khả năng đáp ứng thông tin của hệ thống thông tin kế toán trong quá trình kinh doanh của doanh nghiệp [1].

\section{THIẾT KẾ NGHIÊN CÚ'U}

Nghiên cứu định tính được tiến hành khảo sát và tham khảo ý kiến của một số chuyên gia là các nhà quản lý tại các doanh nghiệp và giảng viên giảng dạy Hệ thống thông tin kế toán tại trường Đại học Công nghiệp TP.HCM, Đại học Kinh tế TP.HCM nhằm nhận diện và xác định các nhân tố ảnh hưởng trong sự phù hợp của hệ thống thông tin kế toán đến hiệu quả hoạt động sản xuất kinh doanh kết hợp với nghiên cứu lý thuyết và các nghiên cứu trước có liên quan. Sau nhiều lần hiệu chỉnh, bảng câu hỏi cuối cùng đã được xây dựng hoàn chỉnh với 42 câu hỏi và đưa vào khảo sát định lượng tại các lớp học Kế toán trưởng, cao học, CFO, CEO ở Thành phố HCM, Đồng Nai, Bình Dương, Long An, Tây Ninh.

Thiết kế thang đo cho bảng hỏi khảo sát: Tất cả các biến quan sát trong các thành phần đều sử dụng thang đo Likert 5 điểm. Thang đo Nhu cầu thông tin đối với hệ thống thông tin kế toán lựa chọn từ 1 đến 5 nghĩa là "hoàn toàn không quan trọng" đến "hoàn toàn rất quan trọng". Thang đo Khả năng đáp ứng nhu cầu thông tin của hệ thống thông tin kế toán lựa chọn từ 1 đến 5 nghĩa là "hoàn toàn không có khả năng đáp ứng" đến "hoàn toàn đủ khả năng đáp ứng toàn diện". Với nhân tố phụ thuộc Hiệu quả hoạt động sản xuất kinh doanh lựa chọn từ 1 đến 5 nghĩa là "hoàn toàn không hiệu quả" đển "hoàn toàn rất hiệu quả".

Thang đo các thành phần (nhân tố): Nhân tố "nhu cầu thông tin đối với hệ thống thông tin kế toán (IR)" được thiết kế đo lường bởi 19 biến quan sát (thang đo). Nhân tố "khả năng đáp ứng $n h u$ cầu thông tin của hệ thống thông tin kế toán (IC)" cũng được thiết kế 19 biến đo lường tương ứng. Nhân tố "sụ phù hợp trong hệ thống thông tin kế toán (AL)" là nhân tố trung gian được kết hợp từ hai thành phần IR và IC. Cuối cùng nhân tố phụ thuộc là "Hiệu quả hoạt dộng kinh doanh của doanh nghiệp $(P)$ " được thiết kế đo lường bởi 4 biến.

Khảo sát định lượng được tiến hành từ đầu tháng 9/2015 đến cuối tháng 3/2016, đối tượng chọn mẫu là các nhà quản lý cấp trung và cấp cao, tức kế toán trưởng hay trưởng phòng kế toán, giám đổc điều hành, giám đốc tài chính. 
Nghiên cứu định lượng này được thực hiện thông qua phương pháp trực tiếp phát phiếu điều tra (bảng hỏi). Phương pháp lấy mẫu phi ngẫu nhiên - lấy mẫu thuận tiện. Mô hình đo lường gồm 42 biến quan sát, theo Hair \&Ctg (1998), kích thước mẫu cần thiết là $n=210(42 \times 5)$. Để đạt được kích thước mẫu đề ra, 250 bảng câu hỏi được gửi phỏng vấn các nhà quản lý. Kết quả thu thập được 243 phiếu phản hồi, trong đó có 11 phiếu trả lời không hợp lệ, còn 232 phiếu đạt yêu cầu. Dữ liệu được nhập và phân tích trên 2 phần mềm SPSS 20 và AMOS 22.

\section{KẾT QUẢ NGHIÊN CÚ̉U \& THẢO LUẬN}

\subsection{Mẫu nghiên cứu}

Kết quả mẫu khảo sát có 232 quan sát (phiếu), trong đó các doanh nghiệp được khảo sát phân nhóm ${ }^{1}$ theo Thời gian hoạt động và Số cán bộ công nhân viên của doanh nghiệp (bảng 1 ). Kết quả cũng cho thấy tỷ lệ các doanh nghiệp nhỏ và vừa chiếm tỷ lệ khá cao $(75.4 \%)$, còn doanh nghiệp quy mô tương đối lớn/lớn chỉ chiếm $24.6 \%$, chủ yếu là các doanh nghiệp hoạt động lâu năm (trên 10 năm). Tuy vậy, mẫu khảo sát vẫn đảm bảo cho nghiên cứu và các kết quả phân tích của mẫu vẫn có ý nghĩa đối với tổng thể.

Bảng 1. Kết quả phân nhóm doanh nghiệp theo thời gian hoạt động và quy mô doanh nghiệp

\begin{tabular}{|c|c|c|c|c|c|c|}
\hline & & & \multicolumn{3}{|c|}{ Quy mô doanh nghiệp (người) } & \multirow{2}{*}{$\begin{array}{l}\text { Tổng } \\
\text { hàng }\end{array}$} \\
\hline & & & Dưới 50 & $\begin{array}{c}\text { Từ } 50 \text { đến } \\
100\end{array}$ & Trên 100 & \\
\hline \multirow{6}{*}{$\begin{array}{l}\text { Thời gian hoạt } \\
\text { động công ty }\end{array}$} & \multirow{2}{*}{ Dưới 5 năm } & Số quan sát & 93 & 18 & 4 & 115 \\
\hline & & Tỷ lệ \% & $80.9 \%$ & $15.7 \%$ & $3.5 \%$ & $100 \%$ \\
\hline & \multirow{2}{*}{ Từ 5 đến 10 năm } & Số quan sát & 32 & 20 & 19 & 71 \\
\hline & & Tỷ lệ \% & $45.1 \%$ & $28.2 \%$ & $26.8 \%$ & $100 \%$ \\
\hline & \multirow{2}{*}{ Trên 10 năm } & Số quan sát & 6 & 6 & 34 & 46 \\
\hline & & Tỷ lệ \% & $13.0 \%$ & $13.0 \%$ & $73.9 \%$ & $100 \%$ \\
\hline \multirow{2}{*}{\multicolumn{3}{|c|}{ Tổng cột }} & 131 & 44 & 57 & 232 \\
\hline & & & $56.4 \%$ & $19.0 \%$ & $24.6 \%$ & $100.0 \%$ \\
\hline
\end{tabular}

Nguồn: tác giả tổng hơp tì̀ SPSS

\section{2. Đánh giá thang đo bằng hệ số tin cậy Cronbach's Alpha và phân tích EFA}

Kết quả kiểm tra độ tin cậy bằng hệ số Cronbach's Alpha cho thấy: 37/42 biến quan sát của các nhân tố là phù hợp (đạt tiêu chuẩn), các nhân tố đều có tương quan với biến tổng lớn hơn 0.3 ; hệ số Cronbach's alpha lớn hơn 0.6 nên có thể kết luận: sau khi loại bỏ 05 biến $^{2}$ quan sát không đạt yêu cầu thì độ tin cậy của các thang đo dùng trong mô hình đảm bảo độ tin cậy cho phép. Trong đó, hai nhân tố là "Nhu cầu thông tin - IR" và "Khả năng đáp ứng nhu cầu thông tin của hệ thống thông tin kế toán - IC" là các nhân tố đa hướng, chính vì vậy việc kiểm tra độ tin cậy Cronbach's Alpha vẫn đảm bảo thực hiện trên từng nhân tố đơn hướng.

Phân tích EFA các nhân tố nghiên cứu: "Nhu cầu thông tin đối với AIS"; "Khả năng đáp ứng nhu cầu thông tin của AIS" và " Hiệu quả hoạt động sản xuất kinh doanh "

Kết quả EFA nhân tố "Nhu cầu thông tin đối với AIS - IR" cho thấy có 2 biến quan sát ban đầu là R01 và $\mathrm{R} 12$ có hệ số tải nhân tố (Factor Loading) nhỏ hơn 0.5 nên bị loại, còn lại 17 biến được nhóm thành 3 thành phần. Nhân tố "Khả năng đáp ứng nhu cầu thông tin của AIS" cho thấy có 3 biến quan sát

\footnotetext{
${ }^{1}$ Phân nhóm doanh nghiệp quy mô vừa và nhỏ ở một số quốc gia khu vực Châu Á theo tiêu chí số nhân viên (Huỳnh Ngọc Hà My, 2014).

${ }^{2}$ Các biến quan sát thuộc IC: báo cáo tổng hợp từ các bộ phận, Thông tin về tình hình thị trường, Thông tin phi kinh tế. Các biến thuộc IR: sự kiện trong tương lai và Mục tiêu chính xác.
} 
ban đầu là $\mathrm{C} 04, \mathrm{C} 11$ và $\mathrm{C} 15$ có hệ số tải nhân tố (Factor Loading) nhỏ hơn 0.5 nên lần lượt cũng bị loại, còn lại 16 biến được nhóm thành 4 thành phần. Cuối cùng, nhóm nghiên cứu đã tiến hành đặt lại tên các thành phần cho phù hợp như sau (bảng 2):

Bảng 2. Tóm tắt thông tin về các thành phần trong phép phân tích

\begin{tabular}{|c|c|c|c|}
\hline Thành phần & $\begin{array}{c}\text { Độ tin cậy } \\
\text { (Cronbach's } \\
\text { Alpha) }\end{array}$ & $\begin{array}{l}\text { Phương sai } \\
\text { trích }\end{array}$ & $\begin{array}{c}\text { Hệ số KMO và } \\
\text { kiểm định } \\
\text { Bartlett }\end{array}$ \\
\hline $\begin{array}{l}\text { R1 - Nhu cầu thông tin liên quan đến các báo cáo } \\
\text { chung phổ biến }\end{array}$ & 0.855 & 67.426 & $\begin{array}{c}0.816 \\
(\mathrm{p}=.000)\end{array}$ \\
\hline $\begin{array}{l}\mathrm{R} 2 \text { - Nhu cầu thông tin phi kinh tế, thông tin phân tích } \\
\text { rủi ro }\end{array}$ & 0.825 & 63.596 & $\begin{array}{c}0.809 \\
(\mathrm{p}=.000)\end{array}$ \\
\hline $\begin{array}{l}\text { R3 - Nhu cầu thông tin liên quan đến các quyết định } \\
\text { kinh doanh }\end{array}$ & 0.886 & 70.116 & $\begin{array}{c}0.842 \\
(\mathrm{p}=.000)\end{array}$ \\
\hline $\begin{array}{l}\text { C1 - Khả năng đáp ứng các thông tin liên quan đến các } \\
\text { quyết định kinh doanh }\end{array}$ & 0.827 & 68.759 & $\begin{array}{c}0.817 \\
(\mathrm{p}=.000)\end{array}$ \\
\hline $\begin{array}{l}\text { C2 - Khả năng đáp ứng các thông tin liên quan đến các } \\
\text { báo cáo chung phổ biến }\end{array}$ & 0.821 & 62.433 & $\begin{array}{c}0.806 \\
(p=.000)\end{array}$ \\
\hline $\begin{array}{l}\text { C3 - Khả năng đáp ứng các thông tin về phân tích rủi } \\
\text { ro và thông tin phi kinh tế }\end{array}$ & 0.815 & 61.599 & $\begin{array}{c}0.789 \\
(\mathrm{p}=.000)\end{array}$ \\
\hline $\begin{array}{l}\text { C4 - Khả năng đáp ứng các thông tin liên quan đến các } \\
\text { vấn đề khác }\end{array}$ & 0.688 & 57.624 & $\begin{array}{c}0.655 \\
(\mathrm{p}=.000)\end{array}$ \\
\hline P - Hiệu quả hoạt động SXKD & 0.914 & 79.895 & $\begin{array}{c}0.850 \\
(\mathrm{p}=.000)\end{array}$ \\
\hline
\end{tabular}

Nguồn: tác giả tổng hơp phân tích tì̀ SPSS

\subsection{Phân tích nhân tố khẳng định $\mathrm{CFA}$}

Kết quả của phép phân tích CFA cho thấy hệ số chưa chuẩn hóa của các biến (37 biến) đều có ý nghĩa thống kê và hệ số đã chuẩn hóa đều lớn hơn 0.5 nên không có biến nào bị loại thêm trong phép phân tích CFA. Cuối cùng ta sử dụng mô hình đo lường gồm 8 thành phần (hình 2) để tiếp tục phân tích mô hình cấu trúc tuyến tính (SEM). Các chỉ số đo độ phù hợp mô hình đều đạt yêu cầu: Chi-square/df = $1.283 ; \mathrm{TLI}=0.937 ; \mathrm{CFI}=0.950$ và $\mathrm{RMSEA}=0.050\left(\mathrm{p} \_\right.$value $\left.=0.000\right)$, nên có thể kết luận mô hình phù hợp với dữ liệu nghiên cứu và đạt được tính đơn nguyên.

Các trọng số chưa chuẩn hóa đều có ý nghĩa thống kê (P-value $<0.05)$, và các trọng số chuẩn hóa đều lớn hơn 0.5 . Vì vậy ta có thể kết luận các biến quan sát dùng để đo lường các thành phần, nhân tố của mô hình đạt được giá trị hội tụ.

Hệ số tin cậy của các thang đo và tổng phương sai trích các thành phần trong mô hình đều đạt tiêu chuẩn (bảng 2). Hơn nữa, ta cũng có hệ số tương quan giữa các thành phần đều nhỏ hơn 0.9. Mặt khác, các giá trị p_value đều nhỏ hơn 0.05 , nên hệ số tương quan của từng cặp thành phần khác biệt so với 1 ở độ tin cậy $95 \%$. Vậy, các thang đo của mô hình đạt giá trị phân biệt. 


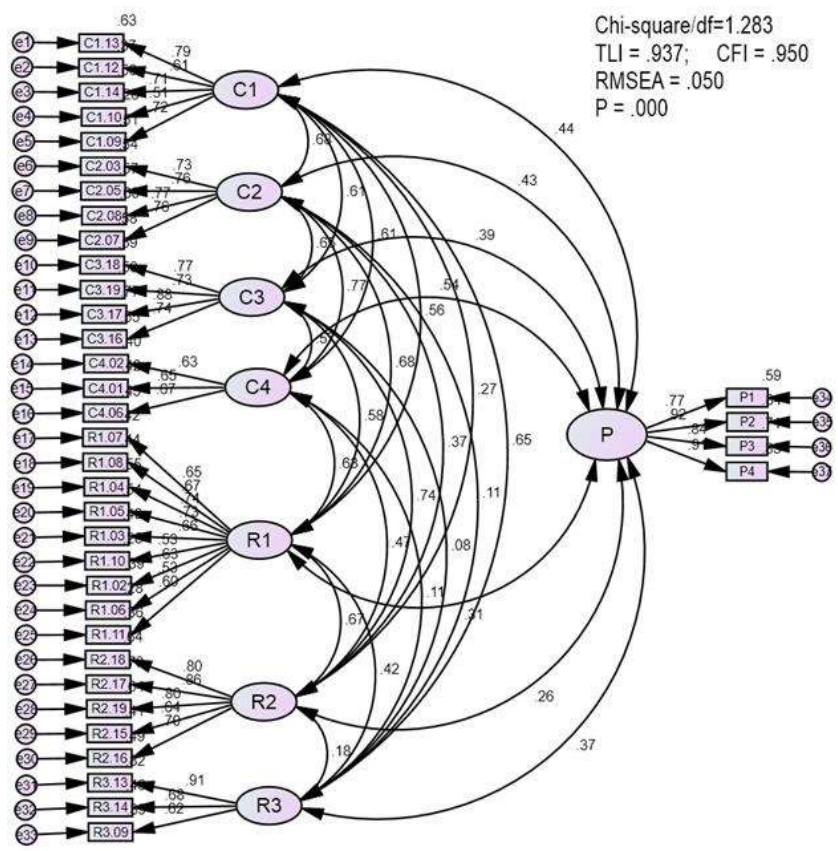

Hình 2. Kết quả phân tích mô hình CFA

Nguồn: tác giả xuất tù phần mềm AMOS 22

\subsection{Phân tích mô hình cấu trúc tuyến tính (SEM)}

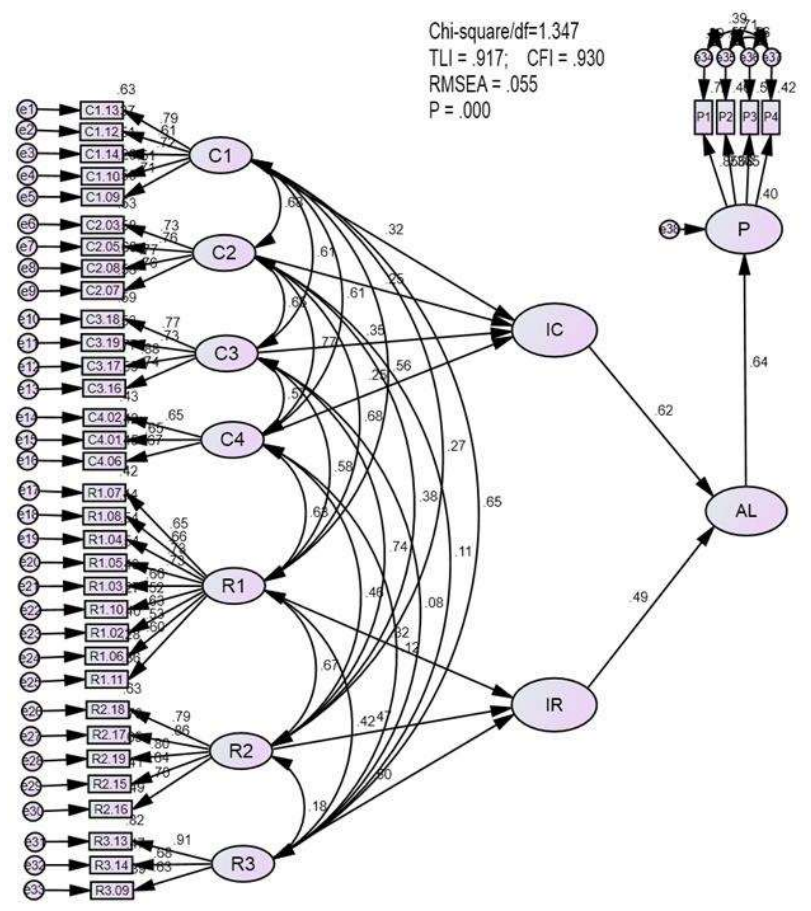

Hình 3. Kết quả phân tích mô hình cấu trúc tuyến tính (SEM) Nguồn: tác giả xuất tù̀ phần mềm AMOS 22 
Tiến hành thiết kế và hiệu chỉnh mô hình cấu trúc tuyến tính (SEM) để phân tích định lượng nhằm kiểm tra và khẳng định mô hình lý thuyết về việc đánh giá mức độ ảnh hưởng sự phù hợp của Hệ thống thông tin kế toán (AL) đến Hiệu quả hoạt động sản xuất kinh doanh của doanh nghiệp $(\mathrm{P})$. Kết quả phân tích thu được như hình 3 . Mô hình có thể nói là phù hợp với dữ liệu nghiên cứu bởi vì Chi-square/df $=1.347, \mathrm{TLI}=0.917, \mathrm{CFI}=0.930 ; \mathrm{RMSEA}=0.055(<0.06)$.

Theo kết quả phân tích SEM, ta có thể diễn giải mô hình như sau:

$$
\left\{\begin{array}{l}
P=0.636 A L+e \\
A L=0.615 I C+0.49 I R \\
I C=0.318 C_{1}+0.248 C_{2}+0.349 C_{3}+0.253 C_{4} \\
\mathrm{IR}=0.322 R_{1}+0.469 R_{1}+0.501 R_{1}
\end{array}\right.
$$

Trong đó, $e$ là sai số (phần dư).

Bảng 3. Trọng số ảnh hưởng của các thành phần trong mô hình SEM

\begin{tabular}{|l|c|c|}
\hline \multicolumn{1}{|c|}{ Thành phần } & $\begin{array}{c}\text { AL - Sự phù họ̣p trong } \\
\text { hệ thống thông tin kế } \\
\text { toán }\end{array}$ & P - Hiệu quả hoạt động SXKD \\
\hline IC - Khả năng đáp ứng nhu cầu thông tin của AIS & 0.615 & 0.391 \\
\hline IR - Các nhu cầu thông tin đối với AIS & 0.490 & 0.312 \\
\hline AL - Sự phù hợp trong hệ thống thông tin kế toán & $X$ & 0.636 \\
\hline
\end{tabular}

*AIS: Hệ thống thông tin kế toán

Nguồn: tác giả tổng hợp phân tích tù SPSS

Qua kết quả bảng 3 , cho thấy các nhu cầu thông tin đối với AIS và khả năng đáp ứng nhu cầu thông tin của AIS đều góp phần tích cực trong sự phù hợp của Hệ thống thông tin kế toán. Cuối cùng, sự phù hợp trong hệ thống thông tin kế toán (AL) có tác động và ảnh hưởng tích cực đến Hiệu quả hoạt động sản xuất kinh doanh của doanh nghiệp $(\mathrm{P})$ với trọng số khá lớn là 0.636 (bảng 3 ).

Kết quả tổng hợp của bảng 4 cho thấy khả năng đáp ưng nhu cầu thông tin của AIS - IC tại các doanh nghiệp khá thấp, mức điểm bình quân của các thang đo là 3.1 và khoảng tin cậy của nó là $(2.4$; 3.8). Trong khi đó, điểm trung bình các thang đo của thành phần IR là 3.6 và khoảng tin cậy là $(2.9 ; 4.2)$ cao hơn IC. Tuy nhiên, kết quả của thành phần IC phù hợp với $\mathrm{P}$ hơn và nhìn chung mức đánh giá là khá thấp. Điều này cũng giải thích vì sao thành phần IC có trọng số trong $\mathrm{AL}$ cao hơn IR (bảng 3).

Bảng 4. Khoảng tin cậy thang đo của các thành phần

\begin{tabular}{|l|c|c|c|c|}
\hline \multicolumn{1}{|c|}{ Thành phần } & $\begin{array}{c}\text { Điểm trung } \\
\text { bình các } \\
\text { thang đo }\end{array}$ & \multicolumn{2}{|c|}{$\begin{array}{c}\text { Độ lệch } \\
\text { chuẩn }\end{array}$} & \multicolumn{2}{|c|}{ Khoảng tin cậy 95\% } \\
\hline IC - Khả năng đáp ứng nhu cầu thông tin của AIS & 3.1 & 0.35 & 2.4 & 3.8 \\
\hline IR - Các nhu cầu thông tin đối với AIS & 3.6 & 0.36 & 2.9 & 4.2 \\
\hline $\mathrm{P}$ - Hiệu quả hoạt động SXKD & 3.2 & 0.081 & 3.0 & 3.4 \\
\hline
\end{tabular}

Nguồn: tác giả tổng hợp và tính toán

Như vậy, nhìn chung năng lực của hệ thống thông tin kế toán (AIS) của các doanh nghiệp Việt nam khá yếu, không đáp ứng đầy đủ nhu cầu thông tin của nhà quản lý (IR), đặc biệt là các báo cáo quản trị. 
Kết quả là hiệu quả hoạt động sản xuất kinh doanh của các doanh nghiệp cũng thấp - chỉ đạt mức trung bình. Dưới đậy là kết quả hệ số của các thành phần trong mô hình mà tác giả đã tổng hợp và tính toán (bảng 5).

Bảng 5. Trọng số ảnh hưởng trực tiếp \& gián tiếp của các nhân tố đến $\mathrm{AL}$ và $\mathrm{P}$

\begin{tabular}{|l|c|c|}
\hline \multicolumn{1}{|c|}{ Thành phần } & $\begin{array}{c}\text { AL - Sự phù hợp } \\
\text { trong hệ thống thông } \\
\text { tin kế toán }\end{array}$ & P - Hiệu quả hoạt động SXKD \\
\hline $\begin{array}{l}\text { R1 - Nhu cầu thông tin liên quan đến các báo cáo } \\
\text { chung phổ biến }\end{array}$ & 0.158 & 0.100 \\
\hline $\begin{array}{l}\text { R2 - Nhu cầu thông tin phi kinh tế, thông tin phân } \\
\text { tích rủi ro }\end{array}$ & 0.230 & 0.146 \\
\hline $\begin{array}{l}\text { R3 - Nhu cầu thông tin liên quan đến các quyết định } \\
\text { kinh doanh }\end{array}$ & 0.245 & 0.156 \\
\hline $\begin{array}{l}\text { C1 - Khả năng đáp ứng các thông tin liên quan đến } \\
\text { các quyết định kinh doanh }\end{array}$ & 0.196 & 0.124 \\
\hline $\begin{array}{l}\text { C2 - Khả năng đáp ứng các thông tin liên quan đến } \\
\text { các báo cáo chung phổ biến }\end{array}$ & 0.153 & 0.097 \\
\hline $\begin{array}{l}\text { C3 - Khả năng đáp ứng các thông tin về phân tích rủi } \\
\text { ro và thông tin phi kinh tế }\end{array}$ & 0.215 & 0.137 \\
\hline $\begin{array}{l}\text { C4 - Khả năng đáp ứng các thông tin liên quan đến } \\
\text { các vấn đề khác }\end{array}$ & 0.156 & \\
\hline Sự phù hợp trong hệ thống thông tin kế toán (AL) & & 0.099 \\
\hline
\end{tabular}

Nguồn: tác giả tổng hợp và tính toán

Kết quả bảng 5 cho thấy sự tác động thuận chiều của các nhân tố trong nhu cầu thông tin kế toán của người dùng $(\mathrm{R} 1, \mathrm{R} 2, \mathrm{R} 3)$ và các nhân tố khả năng đáp ứng nhu cầu thông tin kế toán của hệ thống thông tin kế toán $(\mathrm{C} 1, \mathrm{C} 2, \mathrm{C} 3, \mathrm{C} 4)$ đến sự phù hợp trong hệ thống thông tin kế toán $(\mathrm{AL})$ ). Kết qủa nghiên cứu cũng cho thấy tác động thuận chiều của sự phù hợp trong hệ thống thông tin kế toán $(\mathrm{AL})$ đến hiệu quả hoạt động sản xuất kinh doanh $(\mathrm{P})$ với trọng số dương khá lớn là 0.636 . Kết quả này khăng định sư phù hợp giữa nhu cầu thông tin kế toán và khả năng đáp ứng nhu cầu thông tin kế toán của hệ thống thông tin kế toán tác động tích cực đến hiệu quả hoạt động sản xuất kinh doanh của doanh nghiệp.

\subsection{Thảo luận}

Sự phù hợp trong Hệ thống thông tin kế toán được đo lường bởi khả năng đáp ứng thông tin (IC) và các nhu cầu thông tin từ hệ thống thông tin kế toán của người dùng hay các nhà quản lý (IR). Khi thành phần IC trong $\mathrm{AL}$ có trọng số cao hơn IR đáng kể thì cho thấy hệ thống quản lý của doanh nghiệp bộc lộ sự yếu kém. Nghĩa là các nhà quản lý doanh nghiệp có nhu cầu quá ít về thông tin hay nói cách khác là nhu cầu về các báo cáo quản trị rất ít (chỉ một số báo cáo thông thường), không có các báo cáo dạng phân tích và đề xuất các giải pháp phát triển hoạt động sản xuất kinh doanh. Tuy nhiên, điều này là một dấu hiệu rất tốt đối với doanh nghiệp có bộ phân kế toán quản trị đủ mạnh và một hệ thống kiểm soát nội bộ hữu hiệu và hiệu quả.

Trường hợp ngược lại là trọng số của IC trong $\mathrm{AL}$ nhỏ hơn IR một cách đáng kể, tất nhiên điều này cho thấy Hệ thống thông tin kế toán của doanh nghiệp là không đáp ứng được nhu cầu thông tin của người dùng và các nhà quản lý.

Việc nghiên cứu sự phù hợp trong Hệ thống thông tin kế toán $(\mathrm{AL})$ là rất cần thiết và hữu ích vì $\mathrm{AL}$ có ảnh hưởng tích cực đển Hiệu quả hoạt động kinh doanh của doanh nghiệp. Tuy nhiên, trong mô hình nghiên chưa xem xét đến mối quan hệ giữa các thành phần IC và IR. Thật sụ̂, nhu cầu thông tin của các 
nhà quản lý và nguời dùng thuờng bị ảnh hưởng bởi khả năng đáp ứng của Hẹ thống thông tin kế toán. Hệ thống thông tin kế toán (AIS) của doanh nghiệp thường có một năng lực về cung cấp thông tin nhất định, căn cú vào đó mà các nhà quản lý và ngườ dùng đưa ra nhǘng yêu cầu về thông tin đối với AIS một cách phù hợp.

\section{KẾT LUẬN VÀ Gợi Ý GIẢI PHÁP}

\subsection{Kết luận}

Đóng góp của nghiên cứu là xây dựng mô hình đo lường sự phù hợp trong hệ thống thông tin kế toán $\mathrm{AL})$ thông qua khả năng đáp ứng thông tin $(\mathrm{IC})$ và Các nhu cầu thông tin (IR) của người dùng. Đánh giá sự ảnh hưởng tích cực của $\mathrm{AL}$ đến Hiệu quả hoạt động kinh doanh của doanh nghiệp $(\mathrm{P})$. Mức độ ảnh hưởng của $\mathrm{AL}$ đến $\mathrm{P}$ bị phụ thuộc vào mức độ tương thích (phù hợp) của hai nhóm nhân tố $\mathrm{IC}$ và IR. Nếu mức độ tương thích của $\mathrm{IC}$ và IR càng cao thì mức độ ảnh hưởng của $\mathrm{AL}$ đến $\mathrm{P}$ càng mạnh và ngược lại. Kết quả mô hình nghiên cứu là cơ sở giúp cho các công ty nhận diện các nhân tố cũng như đánh giá mức độ ảnh hưởng của các nhân tố đến Hiệu quả hoạt động kinh doanh của doanh nghiệp trong mô hình nghiên cứu. Doanh nghiệp thực hiện xây dựng hay phát triển Hệ thống thông tin kế toán nhằm nâng cao hiệu quả hoạt động sản xuất kinh doanh thì về cơ bản phải bám sát mô hình cấu trúc tuyến tính trên (hình 3 ) để có thể đưa ra quyết định và các giải pháp phù hợp nhất.

\subsection{Gợi ý một số giải pháp phát triển hệ thống thông tin kế toán cho doanh nghiệp}

Như kết quả nghiên cứu ở trên, sự phù hợp giữa nhu cầu thông tin và khả năng đáp ứng thông tin của hệ thống thông tin kế toán có tác động tích cực đến hiệu quả hoạt động của doanh nghiệp. Thực tế cho thấy các doanh nghiệp có sự phù hợp tốt giữa nhu cầu thông tin kế toán và khả năng đáp ứng thông tin của hệ thống thông tin kế toán sẽ mang lại hiệu quả hoạt động kinh doanh tốt hơn cho doanh nghiệp. Dựa trên kết quả nghiên cứu tác giả đề xuất một số giải pháp nhằm nâng cao sự phù hợp trong hệ thống thông tin kế toán cũng như hiệu quả của hệ thống thông tin kế toán, định hướng đối với các doanh nghiệp chuẩn bị ứng dụng hoặc mong muốn cải thiện hệ thống thông tin kế toán.

5.2.1. Đối với các doanh nghiệp chuẩn bị áp dụng công nghệ thông tin vào việc tổ chức hệ thống thông tin kế toán

Các doanh nghiệp chuẩn bị áp dụng công nghệ thông tin vào việc tổ chức hệ thống thông tin kế toán cần xem xét thận trọng nhu cầu thông tin trong doanh nghiệp trước khi tổ chức hệ thống thông tin kế toán trong môi trường cồng nghệ thông tin, nhằm xác lập phương án cụ thể để có thể tổ chức hệ thống thông tin kế toán một cách thích hợp và hiệu quả. Do đó, các doanh nghiệp cần quan tâm đến các nhu cầu thông tin kế toán nhằm xây dựng một hệ thống thông tin kế toán phù hợp, có khả năng đáp ứng nhu cầu thông tin cần thiết, cung cấp thông tin hữu ích cho nhà quản trị, giúp nhà quản trị đưa ra những quyết định kinh doanh đúng đắn, hiệu quả. Doanh nghiệp có thể tham khảo thực hiện một số bước như sau:

Xây dựng kế hoạch phát triển hệ thống thông tin kế toán đầy đủ, toàn diện và chi tiết về tất cả các vấn đề định hướng, phát triển hệ thống thông tin kế toán theo: mục tiêu phát triển, nhân sự, thời gian, chi phí và tiểu chuẩn chọn lựa nhà cung cấp, tư vấn triển khai. Nhiều doanh nghiệp có thể lựa chọn yếu tố chi phí là hàng đầu nhưng thực tế đã khẳng định chi phí không quan trọng bằng chất lượng về khả năng đáp ứng mà hệ thống thông tin kế toán mang lại.

Trong quá trình triển khai ứng dụng hệ thống thông tin kế toán, cần tuân thủ triệt để quy trình phát triển hệ thống, không rút ngắn giai đoạn phát triển một cách không phù hợp. Cần thiết lập ban quản lý dự án phần mềm, nhằm hạn chế những sai sót trong quyết định của người quản lý thông qua sự tham gia tư vấn của các bộ phận chuyên môn.

Thường xuyên cập nhật các thông tin về công nghệ và ứng dụng công nghệ mới. Trao đổi và nâng cao kiến thức về công nghệ thông tin cũng như tìm hiểu các ứng dụng phần mềm kế toán, phần mềm quản lý. Ngoài ra, để hạn chể rủi ro liên quan đến quyết định của nhà quản lý thì cần chú trọng tới hoạt động giám sát, kiểm soát nội bộ, kiểm toán độc lập. 


\subsection{2. Đối với các doanh nghiệp đã tổ chức hệ thống thông tin kế toán}

Các doanh nghiệp đã tổ chức hệ thống thông tin kế toán nhưng không thành công và đang cần tổ chức lại hệ thống thông tin kế toán, vì hệ thống thông tin kế toán đang sử dụng không đáp ứng được nhu cầu thông tin cho các cơ quan quản lý chức năng hay hệ thống thông tin kế toán đầu tư quá cao so với nhu cầu thông tin thấp. Nhà quản lý cần chú ý phân tích và khắc phục một số vấn đề sau: phân tích ưu và nhược điểm trong việc đáp ứng nhu cầu thông tin của hệ thống thông tin kế toán hiện tại; phân tích mức độ phù hợp giữa những nhu cầu thông tin kế toán mà doanh nghiệp cần và khả năng đáp ứng của hệ thống thông tin kế toán hiện tại đang hoạt động trong doanh nghiệp; phân tích mức độ kiểm soát trong hệ thống thông tin kế toán; phân tích mức tăng, giảm của hiệu quả hoạt động trước và sau khi áp dụng hệ thống thông tin kế toán...Từ đó, doanh nghiệp có thể tiến hành cải thiện lại hệ thống thông tin kế toán hợp lý; hoặc nâng cao trình độ, kỹ năng nhân viên để có thể sử dụng, phân tích những thông tin hữu ích khác mà hệ thống thông tin kế toán được đầu tư cao mang lại, nhằm mang đến hiệu qủa kinh doanh tốt hơn cho doanh nghiệp. Việc đánh giá lại hệ thống thông tin kế toán hiện tại về mức độ đáp ứng yêu cầu thông tin, yêu cầu quản lý, yêu cầu kiểm soát của doanh nghiệp trong sự thay đổi không ngừng nhằm phát triển hay điều chỉnh hệ thống thông tin kế toán trong tương lai như thế nào cho hợp lý, doanh nghiệp cần thực hiện xem xét lại một số điều sau:

Liên quan đến dữ liệu đầu vào của hệ thống thông tin kế toán: cần xác định các dữ liệu nào cần thiết liên quan đến các thông tin nào mà hệ thống thông tin kế toán cần cung cấp cho các đối tượng sử dụng thông tin bên trong hay bên ngoài doanh nghiệp; xác định mức độ đầy đủ và thỏa mãn, thường xuyên của các dữ liệu đầu vào trong quá trình nhập liệu của kế toán, nhược điểm hệ thống chứng từ đang áp dụng,..

Liên quan đến quá trình xử lý dữ liệu trong hệ thống thông tin kế toán: cần xem xét mức độ xử lý dữ liệu, khả năng tập hơp và phân tích dữ liệu, khả năng cập nhật các thay đổi trong chính sách, quy định liên quan ...

Liên quan đến đầu ra của hệ thống thông tin kế toán: cần xem xét nội dung các thông tin đã được xử lý và cung cấp, thời điểm cung cấp báo cáo đã đáp ứng ở mức độ nào; mức độ thỏa mãn yêu cầu cung cấp kết xuất đầu ra, hệ thống báo cáo, phân tích kế toán được thiết lập và cung cấp đáp ứng như thế nào yêu cầu của người sử dụng, mức độ thỏa mãn trong hình thức trình bày báo cáo có phù hợp với đối tượng sử dụng thông tin không, nhược điểm nếu có là gì, mức độ thường xuyên trong hoạt động kiểm tra các kết xuất đầu ra của hệ thống thông tin kế toán như báo cáo, sổ kế toán như thế nào...

Liên quan đển vấn đề lưu trữ trong hệ thống thông tin kế toán: cần xem xét cách thức lưu trữ dữ liệu và thông tin trong hệ thống thông tin kế toán được thực hiện như thế nào, mức độ thuận lợi trong hoạt động tiếp cận, tìm kiếm khi cần dữ liệu và thông tin như thế nào, nhược điểm trong lưu trữ dữ liệu và thông tin của hệ thống thông tin kế toán.

Liên quan đến tổ chức bộ máy kế toán trong hệ thống thông tin kế toán: cần xem xét bộ máy kế toán hiện tại của doanh nghiệp đã đáp ứng như thế nào yêu cầu xử lý và cung cấp thông tin kể toán, doanh nghiệp có chính sách cập nhật và nâng cao trình độ cho nhân viên kế toán bằng những biện pháp nào, bộ máy kế toán tồn tại nhược điểm gì.

Liên quan đển kiểm soát trong hệ thống thông tin kế toán: cần xem xét mức độ hỗ trợ trong kiểm soát quá trình nhập liệu nhằm giảm thiểu sai sót, gian lận; mức độ giám sát, kiểm tra các hành vi truy cập hệ thống; mức độ phân chia trách nhiệm giữa các nhân viên kế toán nhằm hỗ trợ công tác kiểm soát trên toàn hệ thống thông tin kế toán, các chính sách về bảo vệ hệ thống máy tính được thực hiện như thế nào, chính sách bảo vệ dữ liệu và thông tin khỏi sự tiếp cận của những người không có thẩm quyền được thực hiện như thế nào, các nhược điểm cần khắc phục liên quan đến chính sách kiểm soát đã được ban hành.

Liên quan đến tổ chức kế toán theo chu trình trong hệ thống thông tin kế toán: cần xem xét hệ thống thông tin kế toán của doanh nghiệp đã tổ chức theo chu trình ở mức độ nào.

\subsection{3. Đối với nhà cung cấp, tư vấn tổ chức hệ thống thông tin kế toán}

Hệ thống thông tin kế toán trong doanh nghiệp phải hướng đến việc thu thập, xử lý và cung cấp thông tin kế toán tài chính và thông tin kế toán quản trị. Với yêu cầu này hệ thống thông tin kế toán sẽ có thể đáp ứng tối đa yêu cầu sử dụng thông tin trong việc ra quyết định kinh tế của nhà quản lý và các đối tượng bên ngoài như khách hàng, nhà cung cấp...Do đó, các nhà cung cấp phần mền cần phân tích và tìm hiểu hoạt động của doanh nghiệp nhằm đảm bảo thiết kế hệ thống thông tin kế toán thích ứng với từng 
quy mô doanh nghiệp, nhằm đáp ứng tối đa yêu cầu thông tin khác biệt của mỗi quy mô doanh nghiệp. Bên cạnh đó, nhà tư vấn cần có đủ kiến thức, kỹ năng, kinh nghiệm nhằm triển khai phần mền cần phân tích, tìm hiểu hệ thống để đưa ra giải pháp phù hợp và cần triển khai, gắn kết giải pháp đó vào hoạt động của doanh nghiệp, thiết lập hệ thống thông tin kế toán cung cấp các nhu cầu thông tin của doanh nghiệp, phù hợp với đặc thù riêng của từng doanh nghiệp.

\section{TÀI LIẸU THAM KHẢO}

[1]. Nguyễn Thị Tố Quyên (2015), Đo liờng mức độ phù hợp của hệ thống thông tin kế toán trong mối quan hệ với hiệu quả hoạt động kinh doanh của các doanh nghiệp tại Thành phố Hồ Chi Minh, Luận văn thạc sĩ, trường Đại học Kinh tế TP.HCM.

[2]. Huỳnh Thị Hồng Hạnh \& Nguyễn Mạnh Toàn (2013), Đánh giá hiệu quả của hệ thống thông tin kế toán. Tạp chi Kế toán \& kiểm toán, số 6, Tr.11-15.

[3]. Nguyễn Bích Liên (2012). Xác định và kiểm soát các nhân tố ảnh hưởng chất luợng thông tin kế toán trong môi truờng ưng dụng hệ thống hoạch định nguồn lực doanh nghiệp (ERP) tại các doanh nghiệp Việt Nam. Luận án Tiến Sỹ kinh tế, trường Đại học Kinh Tế TP Hồ Chí Minh

[4]. Abernethy, M. A., \& Guthrie, C. H. (1994). An empirical assessment of the "fit" between strategy and management information system design. JournalAccounting \& Finance, 34(2), 49-66.

[5]. Noor Azizi Ismail, Malcolm King (2005), Firm performance and AIS alignment in Malaysian SMEs, International Journal of Accounting Information Systems, 6 (2005) 241-259

[6]. Amidu, M. and Abor, J. (2005), Accounting Information and Management of SMEs in Ghana, African Journal of Finance and Management, Vol.14(1) 2005: 15-24.

[7]. Bollen, K. A (2004), Structural equations with latent variables. John Wiley \& Sons.

[8]. Bolon, D. S. (1998). Information processing theory: REQlications for health care organisations. International Journal of Technology Management, 15(3), 211-221.

[9]. Cragg, P., King, M., \& Hussin, H. (2002). IT alignment and firm performance in small manufacturing firms. The Journal of Strategic Information Systems, 11(2), 109-132.

[10]. Gul FA. The effects of management accounting systems and environmental uncertainty on small business managers' performance. Account Bus Res 1991;22(85):57 - 61 .

[11]. Gul FA, Chia YM (1994), The effects of management accounting systems, perceived environmental uncertainty and decentralisation on managerial performance: A test of three-way interaction. Accounting, Organizations and Society, 19(4/5):413 - 26.

[12]. Raymond L, Pare G, Bergeron F (1995), Matching information technology and organisational structure: An empirical study with implications for performance, European Journal of Information Systems, 4:3- 16.

[13]. Fuller T (1996), Fulfilling IT needs in small businesses: A recursive learning model, International Small Business Journal, 14(4):25 -44.

[14]. Chan YE, Huff SL, Barclay DW, Copeland DG (1997), Business strategic orientation, information systems strategic orientation and strategic alignment. Information Systems Research, 8(2):125-50.

Ngày nhận bài: 24/04/2017

Ngày chấp nhận đăng: 28/10/2017

C 2017 Trường Đại học Công nghiệp thành phố Hồ Chí Minh 